\title{
Os diferentes significados da certificação conferida ao Brasil como estando livre da doença de Chagas
}

\author{
The various meanings of Brazil's certification \\ as free of Chagas disease
}

Alberto N. Ramos Jr. 1

Diana Maul de Carvalho 1

\footnotetext{
1 Núcleo de Estudos de Saúde Coletiva, Centro de Ciências da Saúde, Universidade Federal do Rio de Janeiro. C. P. 68037 , Rio de Janeiro, RJ 21941-590, Brasil. novaes@iis.com.br dianamaul@nesc.ufrj.br
}

Abstract The article discusses Brazil's recent certification as free of Chagas disease transmission by Triatoma infestans, analyzing the various meanings ascribed to this position. Resulting mainly from measures by both the Chagas Disease Control Program (PCDCh) established in Brazil in 1975 and the Southern Cone Initiative launched in 1991, this certification has been interpreted in ways that lead to confusi on between the elimination of Chagas disease transmission by $T$. infestans and eradication of the disease. The present status of vector transmission control in Brazil is discussed, with emphasis on the Northeast, in most States of which T. infestans is not the main species involved in transmission. The article highlights the need to broaden the discussion of the readings and consequences involved in the present control achievements in light of possible harm from misinterpretations that might jeopardize further efforts to control the disease.

Key words Chagas Disease; Vector Control; Triatoma infestans

Resumo Discutem-seas diferentes repercussões da certificação concedi da em março de 2000 a al guns estados do Brasil, em virtude da eliminação da transmissão da doença de Chagas pelo Triatoma infestans. Resultado, em grande parte, das ações do Programa de Controle da Doença de Chagas (PCDCh), estruturado no Brasil a partir de 1975, e das ações definidas pela Iniciativa dos Países do Cone Sul, em 1991, o si gnificado desta certificação foi incorporado de diferentes formas. A questão principal discutida no artigo é a confusão estabel eci da entre eliminação da transmissão vetorial da doença de Chagas pelo T. infestans e erradicação da doença de Chagas. Discute-se o controle vetorial da endemia chagásica no País, em especial na Regi ão Nordeste, onde o T. infestans não representa a principal espécie vetora na maioria dos seus estados. Ressaltase a importância de discutir amplamente a questão do controle dessa endemia no Brasil, no sentido de evitar que informações inapropriadas sejam di fundidas, confundindo a opinião pública, com possí veis prejuízos para o efetivo controle da doença de Chagas.

Palavras-chave Doenças de Chagas; Controle deVetores; Triatoma infestans 


\section{Introdução}

Mais de noventa anos após os primeiros olhares sobre a endemia chagásica (Chagas, 1909), discute-se a possibilidade de se efetivar o seu controle em alguns países da América Latina. Nos últimos dez anos, os resultados das iniciativas regionais que visam eliminar a endemia chagásica na América Latina e que incluem países do Cone Sul, da América Central e andinos vêm levantando essa possibilidade. $O$ foco de ação tem se voltado para a prevenção da transmissão da doença mediante o controle dos bancos de sangue e das populações de vetores. $\mathrm{O}$ grande mérito dessas iniciativas refere-se à mobilização de diferentes países para o controle conjunto da endemia.

No Brasil, as ações de controle foram implementadas de forma efetiva a partir de 1975, quando foi instituído o Programa de Controle da Doença de Chagas (PCDCh) pela Superintendência de Campanhas de Saúde Pública (SUCAM), atual Fundação Nacional de Saúde (FUNASA), dando início à fase de reconhecimento da endemia. Entre 1975 e 1983, foram realizados os primeiros inquéritos entomológico e sorológico, por amostragem, em todo o País (Camargo et al., 1984; Silveira et al., 1984). Desde então, o controle da transmissão vetorial pelo Triatoma infestans, considerado o mais importante vetor do Trypanosoma cruzi no Brasil, é prioritário para o PCDCh (FUNASA, 1994; Silveira et al., 1984; Silveira \& Vinhaes, 1999).

As ações de controle foram direcionadas basicamente ao desenvolvimento de atividades de borrifação com inseticidas e de pesquisas anuais de triatomíneos nos domicílios (Diotaiuti et al., 1998; FUNASA, 1994; Silveira \& Rezende, 1994). Foram priorizadas as áreas consideradas, de acordo com os inquéritos, de maior risco. A área endêmica reconhecida correspondia, em 1983, a 36\% do território nacional, com triatomíneos domiciliados em 2.493 municípios, 50,1\% do total de 4.974 municípios brasileiros (Camargo et al., 1984; Silveira et al., 1984). Em relação às outras áreas, as ações de controle foram de difícil implementação pela presença de outros vetores naturalmente encontrados, considerados de importância secundária (FUNASA, 1994).

A Iniciativa dos Países do Cone Sul, iniciada em 1991 e da qual o Brasil faz parte, comprometeu-se em buscar a eliminação do T. infestans. Definiram-se, ainda, como objetivos para o controle da endemia chagásica nestes países, a redução e a eliminação da transmissão por transfusão sangüínea através da triagem adequada dos potenciais doadores e do fortaleci- mento da rede de bancos de sangue (OPAS, 1992). Paralelamente, chamava-se a atenção para a presença de espécies "não introduzidas" sem definir, entretanto, as metas para o seu controle. Deixava-se clara a menor importância dada a estes triatomíneos, pela decisão de que a redução e/ ou a eliminação da infestação domiciliar destas outras espécies seria empreendida apenas nas áreas onde se observava também a presença do T. infestans (OPAS, 1992).

Como resultado dessa iniciativa, o Uruguai, em 1997, e o Chile, em 1999, foram certificados como estando livres da transmissão da doença de Chagas (WHO, 1998, 2000a, 2000c). O Brasil, por sua vez, encontra-se em pleno processo de avaliação para receber sua certificação, discutida, principalmente, durante as reuniões realizadas em 1999 e 2000.

Em relação ao controle da transmissão transfusional do Tr. cruzi, muito se tem avançado, principalmente a partir de meados da década de 90. As perspectivas de um controle efetivo são boas para os países do Cone Sul, sendo a situação mais preocupante a da Bolívia (Dias \& Schofield, 1998).

O principal objetivo deste artigo é discutir as repercussões no Brasil dessas últimas reuniões da Comissão Intergovernamental do Cone Sul, analisando-se as publicações oficiais e as notícias veiculadas com base nestas reuniões e em seus resultados, com ênfase na prioridade dada à questão do controle vetorial da endemia. De forma a complementar a discussão, faz-se um breve relato sobre a realidade atual da doença de Chagas na Região Nordeste do Brasil, onde, na maioria dos estados, o T. infestans não representa o principal vetor da doença. Por fim, ressalta-se a necessidade de discutir a questão do controle da doença de Chagas no País, considerando-se os determinantes de sua ocorrência em diferentes contextos.

\section{Os diferentes significados}

A descrição e a explicação da situação de saúde-doença dependem, certamente, de quem é o ator social responsável por tal descrição e explicação (Castellanos, 1990).

Apesar de se reconhecerem os determinantes sociais e econômicos da endemia chagásica, poucos estudos têm sido desenvolvidos a fim de trabalhar diferentes realidades locais e os fatores determinantes de sua ocorrência (Silva, 1999). Dessa forma, seguindo as orientações técnico-científicas de experts e de organizações internacionais, estruturaram-se os 
programas de controle da endemia chagásica, voltados basicamente para o controle vetorial.

Durante o VIII Encontro da Comissão Intergovernamental da Iniciativa do Cone Sul, em Tarija, Bolívia, em março de 1999, considerando os dados epidemiológicos e entomológicos disponíveis, discutiu-se a possibilidade de o Brasil ser certificado como estando livre da transmissão vetorial e transfusional da doença de Chagas no ano 2000 (WHO, 1999). As referências para as discussões foram os dados de prevalência da infecção pelo Tr. cruzi em menores de 14 anos até 1998, que mostrariam evidência da interrupção da transmissão da doença de Chagas pelo T. infestans (Silveira \& Vinhaes, 1999). Na publicação da Organização Mundial da Saúde (OMS) intitulada: Chileand Brazil to be Certified Free of Transmission of Chagas Disease(WHO, 1999), pode-se observar a grande importância dada a estes resultados:

"If these results are confirmed, this would be a major accomplishment in the history of public health" (WHO, 1999:10).

Em março de 2000, durante o IX Encontro da Comissão Intergovernamental da Iniciativa do Cone Sul, realizado na Fundação Oswaldo Cruz (FIOCRUZ), no Rio de Janeiro, a FUNASA apresentou duas propostas. A primeira, para que fosse considerada interrompida a transmissão vetorial da doença de Chagas pelo T. infestans nos estados de Goiás, Mato Grosso, Mato Grosso do Sul, Paraíba, Rio de Janeiro e São Paulo; e a segunda, para que fosse examinada a situação epidemiológica dos estados de Minas Gerais, Pernambuco, Piauí e Rio Grande do Sul, com vistas a considerar também interrompida a transmissão da doença de Chagas por aquele vetor (FUNASA, 2000; WHO, 2000b, 2000d), pouco sendo discutido a respeito da participação de espécies de importância “secundária”.

Como desfechos desse encontro, confirmou-se que, dos 12 estados brasileiros onde a presença do T. infestans fora registrada, seis deles (Goiás, Mato Grosso, Mato Grosso do Sul, Paraíba, São Paulo, Rio de Janeiro) estavam livres da transmissão da doença de Chagas por este vetor. Além disso, sugeriu-se que quatro estados (Rio Grande do Sul, Minas Gerais, Piauí e Pernambuco) deveriam receber essa certificação até o final de 2000 (WHO, 2000b, 2000d). Atualmente, as atividades de controle estão sendo direcionadas e intensificadas para os dois estados restantes, Bahia e Tocantins (FUNASA, 2000; WHO, 2000d). Interessante notar que essa informação foi publicada da seguinte forma:

“...confirmed that six of the twelve endemic states in Brazil are free of vectorial transmission of Chagas disease and that a further four states will become free during this year" (WHO, 2000b:154).

Esta publicação da OMS, intitulada Brazil to be Declared Free of Chagas Disease (WHO, 2000b), traz de forma sucinta os resultados daquela reunião e conclui que:

"This result confirms the interruption of vectorial transmission of Chagas diseasein Brazil" (WHO, 2000b:14).

Apesar da ênfase dada pela OMS, em diferentes publicações, sobre a eliminação da transmissão vetorial da doença de Chagas no País, a FUNASA divulga uma nota (FUNASA, 2000), em março de 2000 , a respeito das questões discutidas durante as reuniões, esclarecendo que os resultados referem-se exclusivamente à eliminação do T. infestans, o que condiz com os atuais conhecimentos sobre a doença e com a metodologia implementada para o seu controle:

"A certificação resulta do compromisso assumido, em 1991, quando o Brasil, junto com os demais países do Cone Sul, comprometeu-sea buscar a eliminação do principal responsável pela transmissão vetorial da doença deChagas no país - o Triatoma infestans. A certificação obti da pela FUNASA está cal cada nos resultados de exaustivas avali ações in locu e de estudos técnico-científicos que vieram sendo realizadosao longo dosanos eque, em maio de 1999, culminaram com a recomendação da Comissão Intergovernamental do ConeSul da Organização Pan-Americana deSaúde(OPAS) de avaliar a situação epidemiológica dos estados brasilei ros em relação à interrupção da transmissão peIo Triatoma infestans no Brasil"'(FUNASA, 2000).

No Brasil, durante a semana de realização da IX Reunião da Comissão Intergovernamental do Cone Sul, diferentes notícias foram veiculadas em importantes meios de comunicação, divulgando o encontro e seus resultados. Jornais de grande circulação tinham como título de suas matérias e manchetes:

Doença de Chagas éErradicada em Seis Estados (Folha de São Paulo, 2000);

O Brasil está Mais Perto deVencer o Mal de Chagas (O Globo, 2000);

A Erradicação do Mal de Chagas (Jornal Extra, 2000:13).

A Folha de São Paulo ainda desenvolve a idéia da "erradicação" da doença de Chagas no Brasil, incluindo um "novo" certificado:

"Sei s Estados brasilei ros receberam pela primeira vez o certificado de erradicação da doença de Chagas, concedido por uma comissão formada por representantes dos países do Cone Sul, da OPAS (Organização Pan-Americana da Saúde) e da OMS (Organização Mundial da Saúde)" (Folha de São Paulo, 2000). 
A partir destas notícias, a oposição ao atual governo brasileiro manifesta-se com um artigo intitulado Deputado Suspeita de Fraude na Erradicação de Doença de Chagas (PT, 2000). Desta forma, além das repercussões geradas pela divulgação de forma distorcida de informações técnico-científicas relacionadas ao controle da doença de Chagas, a confusão se estendeu para o lado político:

“...mais um gol pe publicitário do governo FHC: o da erradicação da doença de Chagas em seis estados brasileiros. Num dos estados onde a doença teria sido erradicada, a Paraíba, em mais de $90 \%$ dos municípios éconstatada a presença do bicho barbeiro, transmissor da doença, além de inúmeros registros da enfermidade. A Organização Pan-Americana da Saúde (OPAS) ea Organização Mundial da Saúde (OMS) chegaram a expedir certificados de erradicação da doença. A suposta erradicação chegou a ser anunciada no jornal Folha de São Paulo em 22 de março deste ano. 'Esses dados são absolutamentefalsos', disse hoje o deputado. ...(o deputado) explica o método que o governo utilizou para 'erradicar' a doença: ‘Eles simplesmente deixaram de cadastrar os casos' " (PT, 2000).

Essas colocações são um reflexo bastante claro das possíveis “interpretações das interpretações". Da forma como foi divulgada a informação inicial pela OMS e pela imprensa no Brasil, certamente existem motivos para esta e outras manifestações, envolvendo diferentes setores da sociedade:

“O parlamentar aguarda as informações quepediu ao Ministério da Saúdesobrea suposta erradicação da doença de Chagas na Paraíba. Desua parte, ele tem outros números: o Sindicato dos M édicos do Estado da Paraíba e a imprensa local confirmam a presença do barbeiro - vetor responsável pela transmissão da doença de Chagas - em 182 municípios do Estado e, na maioria deles, com registro detransmissão da doença. Destes, somentenove municípios estão recebendo a cobertura de programas de combate ao inseto da Fundação Nacional de Saúde. 'Como é que el es di vulgam uma informação dessas?', questiona o deputado. Eleinforma que em várias regiões de seu Estado a doença está fora de controle" (PT, 2000).

\section{A transmissão da doença de Chagas no nordeste brasileiro}

Seguindo a discussão sobre a "erradicação" da doença de Chagas no Brasil, a Folha de São Paulo, na sua edição de 22/ 03/ 2000, traz uma entrevista com o Dr. João Carlos Pinto Dias,
Coordenador do Centro de Pesquisas René Rachou, da FIOCRUZ em Belo Horizonte, em que é abordada a questão do T. infestans, tomando-se como exemplo a situação do Nordeste:

“'No Nordeste é onde émais fácil o combate, pel o pouco tempo de existência (do vetor) e a conseqüente frágil adaptação ao meio', explicou. 'Por causa disso a Paraíba conseguiu erradicar a doença no período de 15 anos'".

Em relação à grande maioria dos estados da Região Nordeste do Brasil, o T. infestans em nenhum momento representou a principal espécie de triatomíneo responsável pela transmissão da doença, em virtude de sua introdução recente, provavelmente nas décadas de 50 e 60 (Lucena, 1970). Isso pode ser confirmado pela intenção da FUNASA em eliminar a transmissão vetorial pelo T. infestans em apenas quatro (Paraíba, Piauí, Pernambuco e Bahia) dos nove estados desta região. Mesmo nestas quatro áreas, vetores naturais da região, como, por exemplo, Triatoma brasiliensis, Triatoma pseudomaculata, Triatoma sordida e PanstrongyIus megistus, acabam ten do uma grande importância na transmissão do Tr. cruzi. Mesmo assim, diz a OMS:

“T. infestans is the most important species responsible for the vectorial transmission of the di sease. It is exclusively domestic and shows the highest parasitic infection rates as compared to other species of triatomine present in some endemic areas, such T. brasiliensis and T. pseudomaculata whose vectorial capacity is much Iower" (WHO, 2000d:154).

Diferentes estudos mostram que, com o sucesso do controle do T. infestans, os estados do Nordeste contribuem com $65 \%$ da atual transmissão da doença no Brasil, sendo o T. brasi liensi s e o T. pseudomaculata as espécies mais freqüentemente capturadas pelo PCDCh nessas áreas (Diotaiuti et al., 1998; Soares et al., 2000). O T. brasiliensis tem uma ampla distribuição geográfica (Alagoas, Bahia, Ceará, Goiás, Minas Gerais, Paraíba, Pernambuco, Piauí, Rio Grande do Norte, Sergipe e Tocantins), sendo um dos mais importantes vetores da Região Nordeste (Costa et al., 1998; Diotaiuti et al., 1996; Soares et al., 2000). Estudos recentes confirmam os dados acima.

Na Paraíba, o primeiro inquérito triatomínico nacional evidenciou poucas áreas com a presença do T. infestans. Reconhecendo-se este triatomíneo como espécie introduzida no País, a sua eliminação realmente era factível em um período relativamente curto, o que justificaria a certificação deste Estado (FUNASA, 2000; WHO, 2000b). Diferentes estudos ratificam os 
achados da década de 50, quando o T. pseudomaculata representava uma das espécies mais importantes juntamente com o T. brasiliensis (Pereira \& Coura, 1987). Entre os anos de 1989 e 1994, o Estado da Paraíba contribui com 50\% (26) das amostras sorológicas reativas em todo o Brasil, tendo sido incluídos municípios com presença maciça de triatomíneos, sem a presença, entretanto, do T. infestans (Silveira et al., 1994; Silveira \& Vinhaes, 1999). Estudo mais recente identificou o T. brasiliensis como a espécie mais importante (Coura et al., 1996).

Para os outros três estados nordestinos em avaliação para a certificação da eliminação do T. infestans, a situação não é diferente. Em Pernambuco, diferentes espécies de triatomíneos silvestres têm sido consistentemente relatadas, tanto no peridomicílio como no intradomicílio, na maioria das vezes, o T. brasiliensi s e o P. megistus (Torquato et al., 1998). Esses dados reafirmam os achados de Arthur Neiva \& Belisario Penna, nos anos de 1911 e 1912, quando percorreram o sudoeste deste estado (Neiva \& Penna, 1916).

Ainda em relação a Pernambuco, durante o primeiro Inquérito Entomológico Nacional, a FUNASA chamou a atenção para o encontro de Triatoma rubrofasciata em localidades rurais nos municípios de Carpina, Nazaré da Mata e Tracunhaém (Silveira et al., 1984). Paralelo a estas discussões, o estado provavelmente será certificado ainda este ano como tendo eliminado o T. infestans como vetor da doença de Chagas (FUNASA, 2000).

No Piauí, as primeiras descrições sobre a doença de Chagas começaram a ser publicadas em meados da década de 70 (Figueiredo et al., 1975a, 1975b), já se tendo indícios da presença da doença em 1818 (Neiva \& Pena, 1916; Silva, 1999).

Os primeiros estudos sobre a distribuição de triatomíneos neste estado foram os de Arthur Neiva \& Belisario Penna, entre 1911 e 1912, que relataram a presença de $\mathrm{T}$. brasiliensis, $\mathrm{T}$. pseudomaculata, T. sordida e P. megistus, alguns infectados pelo Tr. cruzi (Neiva \& Penna, 1916). Estudo realizado em 1975 avaliando 28 municípios caracterizou a distribuição geográfica de sete espécies de triatomíneos (T. brasiliensis, T. pseudomaculata, T. sordida, P. megistus, Panstrongylus geniculatus, Rhodnius neglectus e Rhodnius pictepes), capturadas em 18 (64\%) destes municípios. O inquérito entomológico no período 1975 a 1983 no Piauí identificou como principais espécies: T. brasiliensis, T. pseudomaculata, T. sordida, P. megistus, T. infestans e Panstrongylus lutzi (Silveira et al., 1984). Esses autores chamam a atenção para o fato de que a presença do T. infestans limitavase a 12 municípios piauienses localizados na região de fronteira com o Estado da Bahia.

Em 1996, estudo realizado nos municípios de Colônia do Piauí e Oeiras identificou a presença de 49 espécimes de T. pseudomaculata e de 576 espécimes de T. brasiliensis, com 32 (5,5\%) infectados pelo Tr. cruzi (Coura et al., 1996). Entre 1993 e 1997, a FUNASA capturou no Estado, como principais espécies: T. brasiliensis, T. pseudomaculata, T. sordida, T. infestanse P. megistus (Silveira \& Vinhaes, 1998).

Resultados ainda não publicados de um estudo do qual participamos, realizado entre 1999 e 2000, no Município de João Costa, Sudeste do Piauí, reafirmam os estudos anteriores. Foram capturados 1.826 triatomíneos, e, dos 1.062 insetos adultos (58,2\%), identificaram-se: 838 (78,9\%) T. brasiliensis, 198 (18,6\%) T. pseudomaculata, $25(2,4 \%)$ T. sordida e um $(0,1 \%)$ P. lutzi. O inquérito sorológico na área avaliou 2.080 pessoas, com uma prevalência preliminar de infecção pelo Tr. cruzi de 9,9\%. Todas as pessoas caracterizadas como reativas tinham idade superior a dez anos. As atuais mudanças na região, relacionadas ao incremento do turismo direcionado ao Parque Nacional Serra da Capivara, vêm trazendo a possibilidade de reativação de antigos focos de transmissão. Paralelamente, observa-se ainda a desestruturação das ações da FUNASA e a pouca iniciativa municipal, tornando a situação da endemia chagásica na região potencialmente crítica. Enquanto isso, o estado aguarda a certificação de eliminação do T. infestans.

Para as áreas fora das ações principais de controle do T. infestans (Ceará, Maranhão, Sergipe, Alagoas e Rio Grande do Norte), a situação também não é diferente. Inquérito triatomínico realizado no Município de Independência, no Ceará (1996 e 1997), evidenciou que $27,4 \%$ das casas estavam infestadas, sendo $10,8 \%$ no intradomicílio. A maioria dos triatomíneos capturados era T. brasiliensis, além de alguns exemplares de T. pseudomaculata. Inquérito sorológico realizado no período definiu a prevalência de infecção pelo Tr. cruzi em $5,7 \%$, com cinco casos em crianças entre 0 e 10 anos, demonstrando a persistência da transmissão vetorial no Nordeste, apesar da eliminação do T. infestans (Diotaiuti et al., 1998). Ainda neste município, estudo analisando 14 anos de controle conclui que a estratégia atual de controle do T. brasiliensis não parece ser muito eficiente (Carneiro, et al., 1998). Outro estudo no Ceará ressaltou novamente a presença do T. brasiliensise do T. pseudomaculata, sendo as espécies mais encontradas, ocupando 
um grande espaço ao colonizarem ambientes silvestres, peridomiciliares e intradomiciliares (Vieira, 1999).

Um fato relatado pela FUNASA no Ceará e que chamou a atenção de pesquisadores foi a existência de grandes focos intradomiciliares do T. pseudomaculata nas favelas de uma área periurbana de Sobral, achado inesperado em relação ao encontro desta espécie no Brasil (Souza, 1999).

No Sergipe, a espécie de triatomíneo mais encontrada foi o T. pseudomaculata. Outros estudos mostram que, apesar de o trabalho desenvolvido pela FUNASA na década de 80 ter resultado em um bom controle da transmissão vetorial, tal ação parece não ter sido mantida na década de 90 , pelo menos neste estado, onde houve a ocorrência de novas infecções por transmissão vetorial nos últimos seis anos (Oliveira et al., 1998).

No Maranhão, estudo recente procurou esclarecer a situação da distribuição dos triatomíneos, descrevendo 15 espécies encontradas, sendo a mais importante o P. megistus (Rebêlo et al., 1998).

Para o Rio Grande do Norte e Alagoas, poucas informações estão disponíveis. Os vetores mais importantes descritos são o P. megi stus, o T. pseudomaculata e o T. brasiliensis (Silveira et al., 1984).

Na Bahia, estado sem previsão para receber a certificação de eliminação do T. infestans, segundo a FUNASA, as ações estão atualmente concentradas na eliminação deste vetor (FUNASA, 2000; WHO, 2000b). Trabalho realizado de 1996 a 1998 em Juazeiro confirmou os achados do primeiro Inquérito Nacional: T. brasiliensis, T. sordida, T. pseudomaculata, R. neglectus (Marchon-Silva et al., 1999). A partir de 1996, alguns trabalhos vêm levantando a possi bilidade de transmissão vetorial ativa na cidade de Salvador pela presença de espécies de triatomíneos altamente susceptíveis à infecção pelo Tr. cruzi, principalmente o Triatoma tibiamaculata, uma espécie encontrada em ecótopos silvestres (Dias-Lima \& Sherlock, 2000).

No Sudoeste deste estado, mais especificamente em Carinhanha, estudo realizado em assentamentos promovidos pelo Movimento dos Trabalhadores Rurais sem Terra (MST) constatou a existência de grande quantidade de T. sordida, tanto no intradomicílio, como no peridomicílio, e de T. pessoai, T. infestans, T. melanocephala, T. brasiliensis e T. pseudomaculata, estes em menores quantidades, ressaltando-se a necessidade de avaliação do risco de emergência de doenças em novas áreas de ocupação (Sherlock, et al., 1998). Estas são situações pou- co estudadas e não previstas pelo PCDCh e peIa Iniciativa do Cone Sul em relação à doença de Chagas.

\section{Discussão}

As interpretações por parte da imprensa e demais atores sociais envolvidos nos levam à discussão de quanto tais informações podem ser responsáveis por uma desmobilização ainda maior de toda a sociedade para um problema que está longe de ser controlado, com significativo prejuízo para as ações de vigilância e controle vetorial, que buscam a participação ativa da população.

À primeira vista, parece que existe, de forma consciente ou não, a incorporação da idéia de que a eliminação do $\mathrm{T}$. infestans representa a erradicação ou o controle efetivo da doença de Chagas no Brasil.

Em relação ao $T$. infestans, é aceito que o centro de sua dispersão na América do Sul foi a região dos vales interandinos da Bolívia (Dujardin, 1998; Forattini, 1980; Silva, 1999; Veizaga et al., 1999). Como espécie de triatomíneo introduzida e encontrada basicamente no ambiente domiciliar no Brasil, seria possível a sua eliminação (Forattini, 1980; Silveira et al., 1984; Silveira \& Rezende, 1994; Silveira \& Vinhaes, 1999).

Ainda hoje na Bolívia, a doença de Chagas persiste como um importante problema de saúde pública, com o T. infestans ocorrendo em sete dos nove departamentos nos quais a Bolívia se divide, correspondendo a $84 \%$ do território (Veizaga et al., 1999). Sendo o T. infestans espécie naturalmente encontrada na Bolívia, torna-se praticamente inatingível o objetivo de eliminação da transmissão vetorial da doença de Chagas por este triatomíneo naquele país (Figueroa, 1998; Moncayo, 1999; Veizaga et al., 1999). Essa situação mantém o risco constante do transporte passivo do T. infestans desta área para outras no continente americano onde sua ocorrência foi reduzida ou não tinha sido relatada ( Veizaga et al., 1999). Como complicador, na Bolívia ainda não estão disponíveis informações em relação aos dados entomológicos ou epidemiológicos para a avaliação do impacto dos programas de controle vetorial. Estes dados estarão, talvez, disponibilizados em 2001, após quatro a cinco anos das atividades de controle continuado e conclusão do inquérito entomológico e sorológico (Moncayo, 1999).

É importante lembrar que, além do aumento da densidade da população humana em determinadas áreas, a mobilidade das pessoas é 
cada vez maior. Com o grande desenvolvimento dos setores de transportes e de turismo, impulsionado pelo estreitamento dos laços econômicos no Mercosul, as fronteiras entre os países estão cada vez mais tênues. Assim, a vigilância em relação à presença do $\mathrm{T}$. infestans deve ser contínua, a despeito do sucesso de seu controle no Brasil, no Chile e no Uruguai (FUNASA, 2000; WHO, 1998, 1999, 2000a, 2000b, 2000c, 2000d).

Existe, ainda, o risco de ocupação de áreas trabalhadas pelas ações de controle, por espécies silvestres de triatomíneos, o que pode levar a mudanças importantes na epidemiologia da doença de Chagas (Dujardin, 1998). Para se ter uma idéia do problema no Brasil, dos cerca de 126 membros da subfamília Triatominaeconhecidos (Borges et al., 1999), 42 já foram identificados no País. Destes, pelo menos trinta foram capturados em diferentes situações e circunstâncias, no ambiente domiciliar (FUNASA, 1994).

Em relação à reinfestação pelas espécies consideradas secundárias, o problema tornase ainda mais complexo, tanto em termos operacionais, quanto pelas técnicas aplicáveis para controle e vigilância entomológica. As técnicas disponíveis para o tratamento químico com efeito residual são de reduzida efetividade para estas espécies (Schofield et al., 1998).

Com a crescente desmobilização do PCDCh no Brasil a partir de meados da década de 80, as ações de vigilância e controle se tornaram menos efetivas, fazendo com que a intradomiciliação dos triatomíneos seja uma possibilidade concreta. A suspensão experimental da borrifação no peridomicílio resultou no aumento do número de unidades domiciliares positivas e de triatomíneos capturados, o que permite concluir que a borrifação peridomiciliar deve ser mantida, principalmente para o controle do tamanho das colônias (Diotaiuti et al., 1998).

Como exemplo, após a eliminação do T. infestans como principal vetor em partes do Estado de Minas Gerais, o T. sordi da e o P. megistus tornaram-se as espécies mais freqüentemente relatadas nas casas rurais, por "invasão" originária dos focos silvestres (Diotaiuti et al., 1995). Em São Paulo, os dados da Superintendência de Controle de Endemias (SUCEN) indicam que a transmissão da doença de Chagas pelo T. infestans foi interrompida no início da década de 70 (Silva, 1999). Entretanto, ressaltase a preocupação de que espécies como T. sordi da eP. megi stus possam ocupar o vazio ecológico determinado pela eliminação do T. infestans e desenvolver um comportamento sinantrópico, contrariando as expectativas mais otimistas de controle efetivo da endemia (Nascimento et al., 1997).

A presença de vetores de importância secundária é também relevante para as ações das iniciativas dos países andinos e da América Central em virtude de o T. dimidiata, um dos vetores mais importantes destas áreas, possuir extensas populações silvestres, peridomésticas e domésticas (Acevedo et al., 2000; Schofield et al., 1998).

Tais achados acabam por revelar de forma surpreendente o quão pequeno é o conhecimento atual a respeito da capacidade de adaptação dos triatomíneos a novas situações ecológicas (Borges et al., 1999; Soares et al., 2000). O ciclo de transmissão silvestre parece ser muito mais complexo do que se assume hoje; nenhuma generalização ou predição pode ser feita a partir de um único estudo enzoótico de parasitos, pois cada ecótopo deve ser considerado como único (Jansen et al., 1999, 2000). É neste senti do que as ações de controle e de certificação devem ser avaliadas de forma clara em cada área endêmica.

Em muitos desses casos, as novas infestações envolvem espécies pouco conhecidas, consideradas de hábitos exclusivamente silvestres. Existem relatos cada vez mais freqüentes em toda a América Latina de outras espécies de triatomíneos estabel ecendo colônias domiciliares (Borges et al., 1999; Costa, 1999; Diotaiuti et al., 1995; Schofield et al., 1998, 1999; Soares et al., 2000).

Torna-se cada vez mais claro, com base nas experiências verificadas em diferentes países, que é possível eliminar as populações de triatomíneos introduzidos, pelas técnicas disponíveis. Contudo, não se pode parar por aí. A próxima fase no controle da doença de Chagas deve considerar o estudo, a monitorização e o controle de espécies consideradas secundárias e já "eliminadas" (Diotaiuti et al., 1996; Dujardin, 1998; Schofield, et al., 1999; Soares et al., 2000).

A transmissão da doença de Chagas pode ser interrompida em uma escala temporária ou limitada, com chance variável de ser reinstalada, a depender das definições estabelecidas pelas ações de vigilância e controle. Como doença enzoótica, não é passível de erradicação, uma vez que infecções acidentais podem ocorrer (Silveira \& Vinhaes, 1999). A eliminação depende, portanto, da manutenção das atividades de controle da doença por tempo indefinido (Tauil, 1998). A transmissão domiciliar pode ser reinstalada a partir de um foco silvestre, possibilitando as condições necessárias para que a sua ocorrência seja mantida (Silveira, \& Vinhaes, 1999). O objetivo das atividades de 
controle da doença de Chagas nos diferentes países endêmicos deve ser a redução da incidência a níveis tão baixos, que a doença deixe de ser problema de saúde pública (Tauil, 1998).

Todavia, o atual processo de descentralização das ações do PCDCh no Brasil (MS, 2000) vem provocando, em algumas áreas, uma completa desestruturação das ações de controle vetorial. Sem os recursos financeiros e técnicos necessários, a reativação de antigos focos e a manutenção dos já existentes torna-se uma realidade muito próxima para diversos municípios no País. É importante lembrar que, além do controle químico de vetores, a outra perspectiva para a redução da transmissão vetorial seria a melhoria das condições de habitação, justificada também pela possibilidade de redução da virulência do Tr. cruzi em termos evolutivos (Ewald et al., 1998).

Certamente, os objetivos a serem alcançados tornam-se verdadeiros desafios, principalmente ao se considerar o aumento das desigualdades sociais em toda a América Latina. Como exemplo de um novo processo a ser considerado dentro dos fatores de reprodução social já conhecidos e que determina o espaço singular da doença de Chagas, surge no final da década de 70 o MST, movimento que toma força a partir da década de 90. Apenas em 1999, foram organizados 538 acampamentos com aproximadamente 70.000 famílias em todo o Brasil, sendo $55 \%$ destes acampamentos na Região Nordeste (MST, 1999), caracterizando uma nova forma de movimento migratório e de ocupação do território.

Tais fatos trazem à tona a necessi dade de se discutir com maior atenção e profundidade a questão do controle da doença de Chagas no Brasil e em outras áreas endêmicas, sempre buscando o envolvimento de toda a sociedade.

Nesse sentido é que se torna de extrema importância o desenvolvimento de métodos capazes de apreender a complexidade dos processos determinantes que envolvem níveis mais gerais da sociedade com a saúde dos indivíduos e dos grupos sociais (Castellanos, 1990, 1991). Somente a partir daí é que se pode pensar na estruturação de programas de controle da doença de Chagas com maior eficácia e eficiência, principalmente quando se passa a reconhecer que as doenças evoluem juntamente com as sociedades em que se inserem (Silva, 1999). Dessa forma, as iniciativas de controle tornar-se-ão mais claras para todos.

\section{Referências}

ACEVEDO, F.; GODOY, E. \& SCHOFIELD, C. J., 2000. Comparison of intervention strategies for control of Triatoma dimidiata in Nicaragua. Memórias do Instituto Oswaldo Cruz, 95:867-871.

BORGES, E. C.; PIRES, H. H. R.; BARBOSA, S. E.; NUNES, C. M. S.; PEREIRA, M. H.; ROMANHA, A. J. \& DIOTAIUTI, L., 1999. Genetic variability in Brazilian triatomines and the risk of domiciliation. Memórias do Instituto Oswaldo Cruz, 94: 371-373.

CAM ARGO, M. E.; SILVA, G. R.; CASTILHO, E. A. \& SILVEIRA, A. C., 1984. Inquérito sorológico da prevalência de infecção chagásica no Brasil, 19751980. Revista do Instituto de Medicina Tropical de São Paulo, 26:192-204.

CARNEIRO, F. C. F.; LIMA, J. W. O. L.; SCHOFIELD, C. J. \& DIOTAIUTI, L., 1998. Inquérito soroepidemiológico para a doença de Chagas em área predominantemente infestada pelo Triatoma brasiliensis. Revista da Sociedade Brasileira de Medicina Tropical, 31:240. 
CASTELLANOS, P. L., 1990. Sobre el concepto de salud-enfermidad: Descripción y explicación de la situación de salud. Boletín Epidemiológico de la Organización Panamericana dela Salud, 10:1-7.

CASTELLANOS, P. L., 1991. Sistemas Nacionales de Vigilancia dela Situación de Salud Según Condiciones de Vida y el Impacto de las Acciones de Salud y Bienestar. Programa Análisis dela Situación de Salud y sus Tendencias. Washington, DC: Organización Panamericana de la Salud/Organización Mundial de la Salud.

CHAGAS, C., 1909. Nova espécie mórbida do homem, produzida por um tripanossoma (Trypanosoma cruzi): Nota prévia. Brazil Médico, 23:161.

COSTA, J., 1999. The synanthropic process of Chagas disease vectors in Brazil, with special attention to Triatoma brasiliensis Neiva, 1911 (Hemiptera, Reduviidae, Triatominae) population, genetical, ecological, and epidemiological aspects. Memórias do Instituto Oswaldo Cruz, 94:239-241.

COSTA, J.; ALMEIDA, J. R.; BRITTO, C.; DUARTE, R.; MARCHON-SILVA, V. \& PACHECO, R. S., 1998. Ecotopes, natural infection and trophic resources of Triatoma brasiliensis (Hemiptera, Reduviidae, Triatominae). Memórias do Instituto Oswaldo Cruz, 93:7-13.

COURA, J. R.; PEREIRA, J. B.; FILHO, F. I. A.; CASTRO, J. A. F.; CUNHA, R. V.; COSTA, W. \& JUNQUEIRA, A. C. V., 1996. Morbidade da doença de Chagas em áreas do sertão da Paraíba e da caatinga do Piauí. Revista da Sociedade Brasileira de Medicina Tropical, 29:197-205.

DIAS, J. C. P. \& SCHOFIELD, C. J., 1998. Controle da transmissão transfusional da doença de Chagas na Iniciativa do Cone Sul. Revista da Sociedade Brasileira de Medicina Tropical, 31:373-383.

DIAS-LIMA, A. G. \& SHERLOCK, I. A., 2000. Sylvatic vectors invading houses and the risk of emergence of cases of Chagas disease in Salvador, State of Bahia, Northeast Brazil. Memórias do Instituto Oswaldo Cruz, 95:611-613.

DIOTAIUTI, L.; BORGES, E. C.; LOROSA, E. S.; ANDRADE, R. E.; CARNEIRO, F. F. C.; FILHO, O. F. F. \& SCHOFIELD, C. J., 1998. Current transmission of Chagas' disease in the State of Ceará, Brazil. Memórias do Instituto Oswaldo Cruz, 93:65-66.

DIOTAIUTI, L.; FILHO, O. F. F.; CARNEIRO, F. F.; SOARES, R. P. P.; ROMANHA, A. J.; PIRES, H. H. R.; BORGES, E. C. \& SCHOFIELD, C. J., 1996. Caracterização da infestação e variabilidade do Triatoma brasiliensis no Nordeste do Brasil. Revista da Sociedade Brasileira de Medicina Tropical, 29:38-39.

DIOTAIUTI, L.; PEREIRA, A. S.; LOIOLA, C. F.; FERNANDES, A. J.; SCHOFIELD, C. J.; DUJARDIN, J. P.; DIAS, J. C. P. \& CHIARI, E., 1995. Inter-relation of sylvatic and domestic transmission of Trypanosoma cruzi in areas with and without domestic vectorial transmission in Minas Gerais, Brazil. Memórias do Instituto Oswaldo Cruz, 90: 443-448.

DUJARDIN, J. P., 1998. Population genetics and the natural history of domestication in triatominae. Memórias do Instituto Oswaldo Cruz, 93:34-36.

EWALD, P. W.; SUSSMAN, J. B.; DISTLER, M. T.; LIBEL, C.; CHAM MAS, W. P.; DIRITA, V. J.; SALLES, C. A.;
VICENTE, A. C.; HEITMANN, I. \& CABELLO, F. 1998. Evolutionary control of infectious disease: Prospects for vectorborne and waterborne pathogens. Memórias do Instituto Oswaldo Cruz, 93:567-576.

FIGUEIRÊDO, P. Z.; CORREIA-LIMA, F. G. \& PORTELLA-NUNES, J. N., 1975a. Doença de Chagas: Primeiros casos autóctones no Estado do Piauí. Revista da Sociedade Brasileira de Medicina Tropical; 9:105-107.

FIGUEIRÊDO, P. Z.; CORREIA-LIMA, F. G. \& PORTELLA-NUNES, J. N., 1975b. Contribuição ao estudo da distribuição geográfica de triatomíneos no Estado do Piauí. Revista da Sociedade Brasileira de Medicina Tropical, 9:197-202.

FIGUEROA, J. P., 1998. Report of the Workgroup on Parasitic Diseases. Bulletin of the World Health Organization, 76:89-93.

FOLHA DE SÃO PAULO, 2000. Doença de Chagas é erradicada em seis estados. Folha de São Paulo, São Paulo, 22 de março de 2000 ४ttp:// www.uol. com.br/fol/geral/ult22032000027.htm>

FORATTINI, O. P., 1980. Biogeografia, origem e distribuição da domiciliação de triatomíneos no Brasil. Revista de Saúde Pública, 14:265-299.

FUNASA (Fundação Nacional de Saúde), 1994. Controle da Doença de Chagas: Diretrizes Técnicas. Brasília: Fundação Nacional de Saúde, Ministério da Saúde.

FUNASA (Fundação Nacional de Saúde), 2000. FUNASA Obtém Certificação Internacional para a Doença de Chagas. 23 de março de 2000 ঝttp:// www.funasa.gov.br/not/not52.htm>.

JANSEN, A. M.; LISBOA, C. V.; PINHO, A. P. S. MARCHEWSKY, R. S.; MANGIA, R. H. R.; CUPOLILLO, E. \& FERNANDES, O., 2000. A ecologia e a complexidade dos ciclos de transmissão do Trypanosoma cruzi na natureza. In: Doença de Chagas - Manual para Experimentação Animal (T. C. Araújo-Jorge \& S. L. Castro, org. ), pp. 33-38, Rio de Janeiro: Editora Fiocruz.

JANSEN, A. M.; PINHO, A. P. S.; LISBOA, C. V.; CUPOLILLO, E.; MANGIA, R. H. \& FERNANDES, O., 1999. The sylvatic cycle of Trypanosoma cruzi : A still unsolved puzzle. Memórias do Instituto Oswaldo Cruz, 94:203-204.

JORNAL EXTRA, 2000. A erradicação do mal de Chagas. Jornal Extra, Rio de Janeiro, 22 de março, Seção Viva Mais, p.13.

LUCENA, D. T., 1970. Estudo sobre a doença de Chagas no Nordeste do Brasil. Revista Brasileira de Malariologia e Doenças Tropicais, 22:3-174.

MARCHON-SILVA, V.; DIOTAIUTI, L.; ALMEIDA, J. R. \& COSTA, J., 1999. Dimensional parameters of the ecotopes, niche and natural infection of sympatric Triatomines at Juazeiro, Bahia, Brazil. Memórias do Instituto Oswal do Cruz, 93:330.

MONCAYO, A., 1999. Progress towards interruption of transmission of Chagas disease. Memórias do Instituto Oswaldo Cruz, 94:401-404.

MS (Ministério da Saúde), 2000. Controle de Endemias. Brasília: MS.

MST (Movimento dos Trabalhadores Rurais sem Terra), 1999. MST em Dados: Dados dos Acampamentos - 1999. 12 de junho de 2000 «ttp:// www.mst. org.br/bibliotec/acampam/acamp99.htm>. 
NASCIMENTO, C.; MARASSÁ, A. M.; CURADO, I. \& PIAZZA, R. M. F., 1997. Encontro de Panstrongylus megistus em ecótopo artificial: Domiciliação ou mera visitação? Revista da Soci edadeBrasileira de Medicina Tropical; 30:333-336.

NEIVA, A. \& PENA, B., 1916. Viagem científica pelo norte da Bahia, sudeste de Pernambuco, sul do Piauí e do norte a sul de Goiás. Memórias do Instituto Oswaldo Cruz, 8:74-224.

O GLOBO, 2000. O Brasil está mais perto de vencer o mal de Chagas. O Globo, $22 \mathrm{M}$ arço. 19 Maio 2000 <http:// www.globo.com/noticias/arquivo/20000322/ >.

OLIVEIRA, C.; SANTOS, E. M.; SANTOS, S. L. O.; MACEDO, J. F. S. \& SILVA, A. M., 1998. Estudo da prevalência da doença de Chagas no Estado do Sergipe. Revista da Sociedade Brasileira de Medicina Tropical, 31:239.

OPAS (Organização Pan-Americana de Saúde), 1992. Documento OPS.PNSP/92-18 sobrea Iniciativa do ConeSul. Washington, DC: Organização Mundial da Saúde.

PEREIRA, J. B. \& COURA, J. R., 1987. Morbidade da doença de Chagas em populações urbanas do sertão da Paraíba. Revista da Sociedade Brasileira de Medicina Tropical, 20:101-107.

PT (Partido dos Trabalhadores), 2000. Deputado suspeita de fraude na erradicação de doença de Chagas. 14 abril. 9 junho 2000 <tttp:// www.pt.org.br>.

REBÊLO, J. M. M.; BARROS, V. L. L. \& MENDES, W. A., 1998. Espécies de Triatominae (Hemiptera: Reduviidae) do Estado do Maranhão, Brasil. Cadernos deSaúdePública, 14:187-192.

SCHOFIELD, C. J.; DIOTAIUTI, L. \& DIAS, J. C. P., 1998. Control of Triatominae in Latin America. Memórias do Instituto Oswaldo Cruz, 93:67-68.

SCHOFIELD, C. J.; DIOTAIUTI, L. \& DUJARDIN, J. P., 1999. The process of domestication in Triatominae. Memórias do Instituto Oswaldo Cruz, 94:375378.

SHERLOCK, I. A.; DIAS-LIMA, A. G. \& CERQUEIRA, E. L., 1998. Risco de emergência de zoonoses em assentamentos no Estado da Bahia. Revista da Sociedade Brasileira de Medicina Tropical, 31:133.

SILVA, L. J., 1999. A Evolução da Doença de Chagas no Estado de São Paulo. São Paulo: Editora Hucitec/ Fundação para o Estudo e Tratamento das Deformidades Craniofaciais.

SILVEIRA, A. C.; FEITOSA, V. R. \& BORGES, R., 1984. Distribuição de triatomíneos capturados no ambiente domiciliar no período 1975/83, Brasil. Revista Brasileira de Malariologia e Doenças Tropicais, 36:15-312.

SILVEIRA, A. C. \& REZENDE, D. F., 1994. Epidemiologia e controle da transmissão vetorial da doença de Chagas no Brasil. Revista da Soci edade Brasileira de Medicina Tropical, 27:11-22.
SILVEIRA, A. C. \& VINHAES, M. C., 1998. Doença de Chagas: Aspectos epidemiológicos e de controle. Revista da Sociedade Brasileira de Medicina Tropical, 31:15-60.

SILVEIRA, A. C. \&VINHAES, M. C., 1999. Elimination of vector-borne transmission of Chagas Disease. Memórias do Instituto Oswal do Cruz, 94:405-411.

SOARES, R. P. P.; EVANGELISTA, L. G.; LARANJA, L. S. \& DIOTAIUTI, L., 2000. Population dynamics and feeding behavior of Triatoma brasiliensis and Triatoma pseudomaculata, main vectors of Chagas disease in northeastern Brazil. Memórias do Instituto Oswaldo Cruz, 95:151-155.

SOUZA, L. C.; FROTA, F. C. C.; SOUZA, J. A.; ZUZA, C. A. S. \& LIMA, J. W. O., 1999. Descrição de um foco urbano de Triatoma pseudomaculata (Hemiptera: Reduviidae: Triatominae) na cidade de Sobral, norte do Ceará. Resultados preliminares. Revista da Sociedade Brasileira de Medicina Tropical, 32:84-85.

TAUIL, P. L., 1998. Controle de agravos à saúde: Consistência entre objetivo e medidas preventivas. Informe Epidemiológico do SUS, 8:55-58.

TORQUATO, G. N.; SANTOS, C. C. D.; MENDONÇA, M. B.; CARVALHO, S. M. S.; KIDO, E. A.; SANTOS, E. S. C. \& ANDRADE, P. P., 1998. Eco-epidemiology of Chagas' disease in Pernambuco: I. Present role of wild triatomines in the transmission of Trypanosoma cruzi. Memórias do Instituto Oswaldo Cruz, 93:331.

VEIZAGA, H. A.; CARVALHO, M. E.; NASCIMENTO, E. M. M.; RODRIGUES, V. L. C. C.; CASANOVA, C. \& BARATA, J. M. S., 1999. Chagas disease in an area of recent occupation in Cochabamba, Bolivia. Revista deSaúde Pública, 33:230-236.

VIEIRA, J. B., 1999. Epidemiologia da doença de Chagas: Dinâmica do peridomicílio. Revista da Sociedade Brasileira de Medicina Tropical, 32:87-88.

WHO (World Health Organization), 1998. Chagas Disease, Uruguay - Interruption of transmission. Weekly Epidemiological Record, 73:1-4.

WHO (World Health Organization), 1999. Chile and Brazil to be certified free of transmission of Chagas disease. TDR News, 59:10.

WHO (World Health Organization), 2000a. Chagas disease interruped in Chile. TDR News, 61:10.

WHO (World Health Organization), 2000b. Brazil to be declared free of Chagas disease. TDR News, 62:14.

WHO (World Health Organization), 2000c. Chagas disease, Chile - Certification of interruption of transmission. Weekly Epidemiological Record, 75:10-12.

WHO (World Health Organization), 2000d. Chagas disease, Brazil - Interruption of transmission. Weekly Epidemiological Record, 75:153-155. 